\title{
Breakfast Consumption and Cardiovascular Risk Factors in University Going Students
}

\author{
Naureen Naeem ${ }^{1, *}$, Shakeel Ashraf ${ }^{2}$ \\ ${ }^{1}$ Department of Home-Economics, Lahore Garrison University, Lahore, Pakistan \\ ${ }^{2}$ Department of Food Science and Human Nutrition, University of Veterinary and Animal Sciences, Lahore, Pakistan
}

Copyright $\odot 2018$ by authors, all rights reserved. Authors agree that this article remains permanently open access under the terms of the Creative Commons Attribution License 4.0 International License

\begin{abstract}
Objective: Present research was designed to find association of breakfast skipping with metabolic syndrome risk factors in university going students. In the light of findings of the research project we can control these risk factors in young age before they develop any signs of metabolic disorders in late stages of age. Method: This study included 200 University students. The only condition that implied for these participants was that they should not be under any regular medication. They were instructed to be in overnight fasting before sampling day. Three day dietary record was taken through well-organized questionnaires. Based on the frequency of breakfast eating habits, objects were divided into three groups that are (1) regular breakfast eaters (all 3 days) (2) often breakfast eaters ( 1 to 2 days) and rare breakfast eaters (all 3 days breakfast skippers). Multiple metabolic risk factors were measured by their respective methods that are BMI, lipid profile, waist circumference, blood pressure, glucose levels Multiple logistic regression methods were used to evaluate association of breakfast intake with metabolic syndrome risk factors. Results: The number of the subjects called as rare, often and regular breakfast diners were $32(16 \%), 50$ $(25 \%)$ and $138(69 \%)$. Frequency of male was higher as compared to female regular breakfast eaters. The average of triglycerides, BMI, cholesterol, LDL and systolic blood pressure was significantly higher in rare breakfast eaters as compared to regular eaters ( $p$ value $<0.001$ ) whereas HDL levels were found lower in rare breakfast eaters as compared to their counterparts. Consequently, breakfast eaters have higher levels of risk factors as compared to regular eaters. The study was designed to evaluate the association between breakfast skipping habit and metabolic syndrome risk factors in young adults. Rare breakfast eaters were found to have more risk factors of metabolic syndromes than regular and often breakfast eaters. Probably, this is the first study in Pakistan to examine associations of breakfast consumption with metabolic syndrome and cardiovascular risk factors in young adults. Future cohort studies using a prospective design could evaluate these findings and investigate potential
\end{abstract}

mechanisms. Conclusively, skipping breakfast is a common behavior among young adults, and is associated with increased risk of metabolic syndrome and other cardiovascular factors. Improvement of habits related to the frequency of breakfast consumption, in the age group under study, could be a valuable, significant and imperative implication to prevent these risk factors.

Keywords Breakfast, Cardiovascular Diseases, Cholesterol Level, Anthropometric Measurements

\section{Introduction}

Breakfast is the meal taken after overnight sleep, it is usually taken before starting daily routines. Importance of breakfast is immense as body is deprived of most nutrients, glucose, vitamins, and minerals after overnight fasting. So it is the most effective time for absorption of most important nutrients. People who start their daily routine with breakfast looks more focused, determined, and fresh as compared to breakfast skippers. Due to immense importance of breakfast many studies had been conducted to find relationship between different parameters like body mass index (BMI), metabolic syndrome, mental stress, diabetes etc.

Metabolic syndrome can be defined as disorder of energy utilization and storage, its diagnosis can be done if three out of five medical conditions occurs at the same time that are abdominal obesity, increased blood sugar level, elevated blood pressure, high serum triglyceride and low HDL levels in serum. These metabolic conditions increase the risk of developing cardiovascular disorders, diabetes and specially heart failure. Following are the signs and symptoms of metabolic syndrome i.e. abdominal obesity, overweight with fat deposition mainly around waist and hip. Some other important signs are increased triglycerides, low HDL, impaired glucose fasting level.

There can be many reasons for skipping breakfast. Some 
common reasons for skipping breakfast can be associated with various lifestyles and physical conditions like fatigue, disturbance in sleep, busy daily schedule, smoking, infrequent exercise, alcohol drinking, over working and even coronary heart disease [1]. Another reason for skipping breakfast may be due to late night dinner which can have complications like metabolic syndrome and elevated levels of protein urea. High energy food intake at dinner can lead risks like obesity and type 2 diabetes $[1 ; 2]$.

Skipping breakfast can have unhealthy effect on diet quality and increase in metabolic disease risks. Group of people who took breakfast rarely, have poor nutritive values throughout the day. They get most of their energy through unhealthy food source (fat containing foods) which can lead to different metabolic syndrome [3].

Every day diet quality greatly reflects the type of breakfast eaten. If breakfast comprises mostly of cereals it can be associated with lower fats and higher concentration of carbohydrates, proteins and fiber. And if some skipped his all breakfast regularly showed J-shaped relationship with elevation in Body Mass Index (BMI). Skipping breakfast due to late night dinner can results in poorer diet quality and habitual skipping which can lead to metabolic syndrome [4].

In cross-sectional analysis breakfast skipping has been associated with increase in BMI in children [5] and adults [6]. Several longitudinal studies revealed the effect of breakfast consumption overtime on weight status and most, but not all, suggest that breakfast skipping is associated with weight gain. Studies with follow-up ranging from 3 years to 10 years suggested that breakfast skipping was associated with weight gain in children with normal weight [5], overweight girls, adolescents, and men. In a controlled trial, obese women randomly assigned to either breakfast eating or no breakfast eating showed no difference in weight loss at the end of the12-wk trial or at the 6-months follow-up [7].

Present study was designed to evaluate the effects of skipping breakfast on age, height, weight, BMI, cardiovascular and metabolic disorders. The purpose of this study was to find association of skipping breakfast in the population of Pakistan due to lot of differences in eating patterns, quality of diet and lifestyle.

\section{Materials and Methods}

\subsection{Research Design and Method}

Two hundred university students from different disciplines and their acquaintances were included in this study. The subjects were between 20 and 25years of age, and were not taking any medications regularly and will not have any family history of hypertension, diabetes and heart attack (annexure 2). The data from subjects was collected through questionnaire (annexure 2) and included in the final analyses. All subjects were provided informed consent before participation.

\subsection{Dietary Assessment}

The 3-day dietary intake over one weekend day and two weekdays was collected from each subject using a $24 \mathrm{Hr}$ recall and a 2-dayDR (Daily record).To increase the accuracy of the $24 \mathrm{HR}$ recall, the subjects were instructed and interviewed in three stages. One day dietary intake was obtained using 24-hour recall method from each subject. Dietary interview was conducted in 3 steps, (i) List of foods eaten during the previous day, (ii) detailed information of all the foods eaten, (iii) the final probing for any items forgotten [8]. Food group intake and the amount of energy and nutrients consumed was calculated for each subject according to Asian Nutrition and Health association and was used in the statistical analysis.

\subsection{Subject Classifications}

Breakfast was defined as the meal eaten in the morning, and any food or beverages consumed in the morning was classified as breakfast. In a previous study more than 2 days of dietary intake data classified breakfast consumption, but subjects were divided into three subgroups according to the frequency of skipping breakfast over the 3-day dietary intake data collection period. Group one included the subjects who skipped breakfast on 2 or more of the 3 days were classified as a 'Rare breakfast eater' because breakfast was skipped on more than $50 \%$ of the days studied. Group 2 included subjects who skipped breakfast on 1 of the 3 days were placed in the 'Often breakfast eater' group, and group 3 included those who did not skip breakfast on any of the 3 days of the study was placed in the 'Regular breakfast eater' group. This particular study had also been conducted in some other populations (Korean and American) and provided the base line for the present research. [8]

\subsection{Biochemical, Anthropometric, and Blood Pressure Measurements}

Blood was collected after an 8 hours fast. Micro lab 300 instrument (Human kit) was used to analyze serum triglycerides (TG), low-density lipid cholesterol (LDL-C), high-density lipid cholesterol (HDL-C) and serum fasting glucose. Systolic and diastolic blood pressure was measured by using sphygmomanometer. Weight and height was measured using extensometer. Body mass index was measured using weight $(\mathrm{kg}) /$ height $(\mathrm{M})$ squared.

\section{Results}

The trend of breakfast in male and female young adults. More youngsters were regular breakfast eaters than breakfast skippers as shown in the following Tables $(1,2)$. 
Table 1. Characteristics of participants according to breakfast intake

\begin{tabular}{|c|ll|ll|}
\hline & $\begin{array}{l}\text { Male } \\
(\mathbf{1 0 9 )}\end{array}$ & $\mathbf{( 5 1 . 9 \% )}$ & $\begin{array}{l}\text { Female } \\
(\mathbf{9 1})\end{array}$ & $\mathbf{( 4 8 . 1 \% )}$ \\
\hline Rare Breakfast Eaters & 22 & $(20 \%)$ & 10 & $(11 \%)$ \\
\hline Often breakfast Eaters & 23 & $(21 \%)$ & 27 & $(30 \%)$ \\
\hline Regular Breakfast Eaters & 64 & $(59 \%)$ & 54 & $(59 \%)$ \\
\hline
\end{tabular}

Table 2. Cardio metabolic values in young adults

\begin{tabular}{|c|c|c|c|c|c|}
\hline & Regular & Often & Rare & p-value & $\begin{array}{c}\text { p-value } \\
\text { for trends }\end{array}$ \\
\hline BMI & 20.7 & 20.4 & 22.8 & $<\mathbf{0 . 0 0 1}$ & $<\mathbf{0 . 0 0 1}$ \\
\hline Cholesterol (mg/dl) & 163.7 & 166.7 & 171.8 & $\mathbf{0 . 1 9}$ & 0.13 \\
\hline HDL(mg/dl) & 43.8 & 43.6 & 41.6 & $\mathbf{0 . 0 1}$ & $<0.001$ \\
\hline LDL(mg/dl) & 87.0 & 103.3 & 92.6 & $\mathbf{0 . 0 6}$ & 0.03 \\
\hline Triglycerides(mg/dl) & 151.5 & 145.3 & 172.5 & $<\mathbf{0 . 0 0 1}$ & $<0.001$ \\
\hline Blood Sugar(mg/dl) & 76.9 & 86.5 & 86.0 & $\mathbf{0 . 1 7}$ & 0.06 \\
\hline Waist Circumference(cm) & 68.62 & 68.52 & 69.02 & $\mathbf{0 . 7 7}$ & 0.57 \\
\hline Systolic BP(mmHg) & 103.39 & 104.06 & 102.61 & $<\mathbf{0 . 0 0 1}$ & 0.02 \\
\hline Diastolic BP(mmHg) & 65.44 & 66.48 & 65.28 & $\mathbf{0 . 0 1}$ & 0.15 \\
\hline
\end{tabular}

\subsection{Breakfast Trend in Males}

Out of the 200 studied subjects 109 (51.9\%) were male and most of them fall in the category of rare breakfast eaters $(68 \%)$ ). frequency of male in often $(46 \%)$ and regular (54\%) breakfast eaters was almost equal to women. It was also observed those young males were regular breakfast eaters as compared to any other category.

\subsection{Breakfast Trend in Females}

A sum of 91 included subjects were females out of the total 200 subjects. It was observed that frequency of females was higher in the often $(59 \%)$ breakfast eaters. Distribution of female in rare $(11 \%)$ and regular $(30 \%)$ breakfast eaters was low as compared to the male. It was also concluded from the present study that adult females are regular breakfast eaters as compared to any other age group.

The present study showed that young rare breakfast eaters have higher BMI values as compared to regular and often breakfast eaters. This study also showed that cholesterol, triglycerides level and waist circumference were significantly increased in rare breakfast eaters as compared to often and regular breakfast eaters. It also showed a slight decrease in HDL levels of rare breakfast levels as compare to other two groups. No significant variation was seen in blood sugar fasting levels among the groups. So these tables showed that metabolic risk factors were more among the rare breakfast eaters.

\subsection{Average BMI of Different Breakfast Frequency Groups}

Present study revealed that BMI of rare breakfast eaters were higher among the rare breakfast eaters as they were compared with other groups of often breakfast eaters and regular breakfast eaters. So regularly breakfast eating proves to be helpful in maintaining healthier BMI levels.

\subsection{Average Cholesterol Levels of Different Breakfast Frequency Groups}

This study revealed that average cholesterol levels were higher in the group that used to eat breakfast rarely. As this trend started decreasing in the groups in which frequency of breakfast was increased.

\subsection{Average HDL Levels of Different Breakfast Frequency Groups}

HDL is considered as healthy cholesterol that reduces the risk of cardiovascular diseases. So according to this study it showed that HDL levels were significantly lower in the group who used to eat breakfast rare as compared to the other group who ate breakfast often and regularly.

\section{Statistical Analysis}

All statistical analyses were performed using the Statistical Package for the Social Sciences 20 (SPSS 20). The distribution of subjects in breakfast consumption groups was evaluated using the $\chi^{2}$ test. The mean and standard differences deviation was calculated for each breakfast consumption group. Mean among groups was evaluated by using a general liner model (GLM). The ORs (odd ratios) and 95\% CIs (confidence interval) for metabolic syndrome risk factors was calculated using logistic regression analysis. 


\section{Discussion}

This study confirmed the association between breakfast frequency and CVD risk factors in samples drawn for young university going students. We found skipping breakfast increased the risk of general and abdominal obesity, metabolic syndrome and having elevated TG, LDL-C and lower HDL-C in the children. Previous studies confirmed that skipping breakfast can lead to abdominal obesity $[9 ; 10]$.In this study we found that there is strong association of metabolic syndrome risk factors with rare breakfast eating habit. When other factors were added to the model, this association remained or was only slightly weakened. Another study reported that rare breakfast eaters had a lower total energy intake but a higher BMI than did breakfast eaters. [11].Another study reported that the usual breakfast eater consumed greater amounts of energy, carbohydrates, and fiber but lower total percentages of total calories from fat. [12].Those that daily eats breakfast also seems much more physically active than breakfast skippers. This dietary profile has the potential to improve energy balance and may improve glucose and insulin parameters and lead to increased satiety and lower weight. [13].These results suggest that although skipping breakfast results in a lower daily energy intake, it increases eating snacks, sedentary lifestyle and cannot be used to control obesity. [14]. In contrast, eating breakfast is associated with increased eating frequency and this may in turn promote less efficient energy utilization by increasing dietary-induced thermogenesis, leading to lower BMI. [15]. A complex interaction of environment and behavioral factors is suggested as the underlying cause of metabolic syndrome. However, the impact of diet pattern on this health problem is inconclusive. [16]. The present research concluded that skipping breakfast significantly increased the risk of having metabolic syndrome and cardiovascular risk factors. Our findings are in line with the results of a study among Australian children that showed that skipping breakfast may have determined the effects on their cardio metabolic health [17;21;22].

Our findings regarding cardiovascular risk factors are supported by the result of a clinical trial. Researchers have found women had higher total cholesterol and LDL-C after skipping breakfast than the ones who ate breakfast $[18 ; 23 ; 24 ; 25 ; 26]$. Another study showed that weekly frequency of eating breakfast was negatively associated with fasting glucose and triglycerides regardless of other variables [19;27;28]. As indicated in the current study, the risk of elevated TG, LDL-C and low HDL-C was significantly increased in subjects who skipped breakfast. In addition, the changes in lipid levels may be due to change in dietary habits which led to a considerable imbalance in food consumption, with low nutrient density characterizing diets among more than one third of the households $[20 ; 29 ; 30 ; 31]$. On the other hand, one of the most important changes in lifestyle is skipping meals, mainly breakfast. It has been reported to be associated with unhealthy behaviors, such as consumption of a poorer diet, and having physical inactivity $[21: 32 ; 33 ; 34 ; 35]$, that can result in higher metabolic syndrome components $[22,36 ; 37,38]$.In prevention and treatment of metabolic syndrome, we might benefit more from regular healthy meals than from skipping meals. Because common breakfast comes from the core food groups, it is a nutritious meal and is low in fat. [23;39]. So, for compensation later during the day, breakfast skippers have higher intakes of fat, energy, and lower intakes of fiber, vitamins and minerals than breakfast eaters[24;40]Interestingly, in our study, the metabolic syndrome components that were more strongly related to skipping breakfast were waist circumference, TG and low HDL-C, which are the well-known predictors of insulin resistance, and CVD risk, so emphasizing the importance of eating breakfast as a possible behavioral pattern for cardio metabolic risk, especially metabolic syndrome[25;41]

\section{REFERENCES}

[1] Kutsuma A, Nakajima K, Suwa K. 2014. Potential association between breakfast skipping and concomitant late-night-dinner eating with metabolic syndrome and proteinuria in the Japanese population. Scientifica. 2014.

[2] De Castro JM. 2009. When, how much and what foods are eaten are related to total daily food intake. Br J Nutr. 102(08): $1228-1237$.

[3] Min C, Noh H, Kang Y-S, Sim HJ, Baik HW, Song WO, Yoon J, Park Y-H, Joung H. 2011. Skipping breakfast is associated with diet quality and metabolic syndrome risk factors of adults. Nutr Res Pract 5(5): 455-463.

[4] Ruxton C, Kirk T. 1997. Breakfast: a review of associations with measures of dietary intake, physiology and biochemistry. Br J Nutr. 78(02): 199-213.

[5] Berkey CS, Rockett H, Gillman MW, Field A, Colditz G. 2003. Longitudinal study of skipping breakfast and weight change in adolescents. Int J Obes. 27(10): 1258-1266.

[6] Berg C, Lappas G, Wolk A, Strandhagen E, Torén K, Rosengren A, Thelle D and Lissner L. 2009. Eating patterns and portion size associated with obesity in a Swedish population. Appetite. 52(1): 21-26.

[7] Schlundt DG, Hill JO, Sbrocco T, Pope-Cordle J, Sharp T. 1992. The role of breakfast in the treatment of obesity: a randomized clinical trial. Am J ClinNutr55(3): 645-651.

[8] Kang H, Jung HJ, Paik HY. 2009. Analysis of foods and nutrients intake obtained at the final probing step in 24-hour recall method. Nutr Res Pract. 42(2): 158-170.

[9] Duncan S, Duncan EK, Fernandes RA, Buonani C, Bastos KD, Segatto AF, Codogno JS, Gomes IC and Freitas IF. 2011. Modifiable risk factors for overweight and obesity in children and adolescents from São Paulo, Brazil. BMC 
Public Health. 11(1): 585.

[10] Dupuy M, Godeau E, Vignes C and Ahluwalia N. 2011. Socio-demographic and lifestyle factors associated with overweight in a representative sample of 11-15 year olds in France: results from the WHO-Collaborative Health Behaviour in School-aged Children (HBSC) cross-sectional study. BMC Public Health. 11(1): 442.

[11] Cho S, Dietrich M, Brown CJ, Clark CA and Block G. 2003. The effect of breakfast type on total daily energy intake and body mass index: results from the Third National Health and Nutrition Examination Survey (NHANES III). Journal of the American College of Nutrition. 22(4): 296-302.

[12] Timlin MT, Pereira MA, Story M and Neumark-Sztainer D. 2008. Breakfast eating and weight change in a 5-year prospective analysis of adolescents: Project EAT (Eating Among Teens). Pediatrics. 121(3): e638-e645.

[13] Júnior IFF, Christofaro DG, Codogno JS, Monteiro PA, Silveira LS and Fernandes RA. 2012. The association between skipping breakfast and biochemical variables in sedentary obese children and adolescents. The Journal of pediatrics. 161(5): 871-874.

[14] Keski-Rahkonen A, Kaprio J, Rissanen A, Virkkunen M and Rose RJ. 2003. Breakfast skipping and health-compromising behaviors in adolescents and adults. European Journal of Clinical Nutrition. 57(7): 842-853.

[15] Drummond S, Crombie N and Kirk T. 1996. A critique of the effects of snacking on body weight status. European Journal of Clinical Nutrition. 50(12): 779-783.

[16] Kelishadi R, Gouya MM, Adeli K, Ardalan G, Gheiratmand R, Majdzadeh R, Mahmoud-Arabi MS, Delavari A, Riazi MM and Barekati H. 2008. Factors associated with the metabolic syndrome in a national sample of youths: CASPIAN Study. Nutrition, metabolism and cardiovascular diseases. 18(7): 461-470.

[17] Smith KJ, Gall SL, McNaughton SA, Blizzard L, Dwyer T, Venn AJ. 2010. Skipping breakfast: longitudinal associations with cardiometabolic risk factors in the Childhood Determinants of Adult Health Study. Am J Clin Nutr. 92(6): 1316-1325.

[18] Farshchi HR, Taylor MA and Macdonald IA. 2005. Beneficial metabolic effects of regular meal frequency on dietary thermogenesis, insulin sensitivity, and fasting lipid profiles in healthy obese women. The American journal of clinical nutrition. 81(1): 16-24.

[19] Ghassemi H, Harrison G and Mohammad K. 2002. An accelerated nutrition transition in Iran. Public health nutrition. 5(1a): 149-155.

[20] Timlin MT and Pereira MA. 2007. Breakfast frequency and quality in the etiology of adult obesity and chronic diseases. Nutrition reviews. 65(6): 268-281.

[21] Sierra-Johnson J, Undén AL, Linestrand M, Rosell M, Sjogren P, Kolak M, Faire U, Fisher RM and Hellénius ML. 2008. Eating meals irregularly: a novel environmental risk factor for the metabolic syndrome. Obesity. 16(6): $1302-1307$

[22] Williams P. 2005. Breakfast and the diets of Australian adults: An analysis of data from the 1995 National Nutrition Survey*. International journal of food sciences and nutrition.
56(1): $65-79$.

[23] Agostoni C, Brighenti F. 2010. Dietary choices for breakfast in children and adolescents. Crit Rev Food Sci Nutr. 50(2): 120-128.

[24] Al-Lawati JA, Mohammed AJ, Al-Hinai HQ and Jousilahti P. 2003. Prevalence of the metabolic syndrome among Omani adults. Diabetes care. 26(6): 1781-1785.

[25] Almoosawi S, Prynne C, Hardy R and Stephen A. 2013. Time-of-day and nutrient composition of eating occasions: prospective association with the metabolic syndrome in the 1946 British birth cohort. International Journal of Obesity. 37(5): 725-731.

[26] Deshmukh-Taskar P, Nicklas TA, Radcliffe JD, O'Neil CE, Liu Y. 2013. The relationship of breakfast skipping and type of breakfast consumed with overweight/obesity, abdominal obesity, other cardiometabolic risk factors and the metabolic syndrome in young adults. The National Health and Nutrition Examination Survey (NHANES): 1999-2006. Public health nutrition. 16(11): 2073-2082.

[27] DAH, Cabili C, Briefel RR, Williams N, Suitor CW. 2008. Summary of published evidence related to the dietary guidelines for children, 2004-2008. Final report submitted to the US Dept of Health and Human Services, Assistant Secretary for Planning and Evaluation. Washington, DC: Mathematica Policy Research

[28] Grundy SM, Hansen B, Smith SC, Cleeman JI and Kahn RA. 2004. Clinical management of metabolic syndrome report of the American Heart Association/National Heart, Lung, and Blood Institute/American Diabetes Association conference on scientific issues related to management. Circulation. 109(4): 551-556.

[29] Kant AK, Andon MB, Angelopoulos TJ, Rippe JM. 2008. Association of breakfast energy density with diet quality and body mass index in American adults: National Health and Nutrition Examination Surveys, 1999-2004. Am J Clin Nutr. 88(5): 1396-1404

[30] Khader Y, Bateiha A, El-Khateeb M, Al-Shaikh A and Ajlouni K. 2007. High prevalence of the metabolic syndrome among Northern Jordanians. Journal of Diabetes and its Complications. 21(4): 214-219.

[31] Odegaard AO, Jacobs DR, Steffen LM, Van Horn L, Ludwig DS and Pereira MA. 2013. Breakfast frequency and development of metabolic risk. Diabetes care. 36(10): 3100-3106.

[32] Shafiee G, Kelishadi R, Qorbani M, Motlagh ME, Taheri M, Ardalan G, Taslimi M, Poursafa P, Heshmat R, Larijani B. 2013. Association of breakfast intake with cardiometabolic risk factors. J Pediatr (Rio J). 89(6): 575-582.

[33] Soga Y, Shirai C and Ijichi A. 2013. [Association between daily lifestyle and the risk of metabolic syndrome among young adults in Japan. An analysis of Kobe city young adult health examination data]. [Nihon koshu eisei zasshi] Japanese journal of public health. 60(2): 98-106.

[34] Suliga E and Kozie D. 2015. Association between dietary patterns and metabolic syndrome in individuals with normal weight: a cross-sectional study. Nutrition Journal. 14(1): 55.

[35] Tajima M, Lee JS, Watanabe E, Park JS, Tsuchiya R, Fukahori A, Mori K and Kawakubo K. 2014. Association 
between changes in 12 lifestyle behaviors and the development of metabolic syndrome during 1 year among workers in the Tokyo metropolitan area. Circ J. 78: $1152-1159$

[36] Uemura M, Yatsuya H, Hilawe EH, Li Y, Wang C, Chiang C, Otsuka R, Toyoshima H, Tamakoshi K and Aoyama A. 2015. Breakfast Skipping is Positively Associated with Incidence of Type 2 Diabetes Mellitus: Evidence from the Aichi Workers' Cohort Study. Journal of Epidemiology. 25(5): 351.

[37] van den Boom A, Serra-Majem L, Ribas L, Ngo J, Pérez-Rodrigo C, Aranceta J and Fletcher R. 2006. The contribution of ready-to-eat cereals to daily nutrient intake and breakfast quality in a Mediterranean setting. J Am Coll Nutr. 25(2): 135-143.

[38] Veltsista A, Laitinen J, Sovio U, Roma E, Järvelin M-R and
Bakoula C. 2010. Relationship between eating behavior, breakfast consumption, and obesity among Finnish and Greek adolescents. Journal of nutrition education and behavior. 42(6): 417-421.

[39] Williams P. 2007. Breakfast and the diets of Australian children and adolescents: an analysis of data from the 1995 National Nutrition Survey. Int J Food Sci Nutr. 58(3): 201-216.

[40] Wennberg M, Gustafsson PE, Wennberg P and Hammarström A. 2015. Poor breakfast habits in adolescence predict the metabolic syndrome in adulthood. Public health nutrition. 18(01): 122-129

[41] Yi BS and Yang IS. 2006. An exploratory study for identifying factors related to breakfast in elementary, middle and high school students. Korean Journal of Community Nutrition. 11(1): 25-38. 